REVIEW ARTICLE

\title{
Artifacts, Pitfalls and Normal Variants
}

\author{
Ivica Zalud, Frederico Rocha
}

\begin{abstract}
Image artifacts are encountered daily in clinical ultrasonography and may be a source of confusion for the interpreting sonologist. The understanding of the mechanisms behind their production allows recognition of artifacts and avoids misinterpretations and incorrect diagnoses. Some artifacts may be avoidable and arise secondary to improper scanning technique. Other artifacts are generated by the physical limitations of the modality.

Recognition of artifacts in everyday work is of great significance for final diagnosis, since their erroneous interpretation not only compromises the value of ultrasound finding, but may also lead to an inadequate therapeutic approach.

The objective of this article is to describe and illustrate many of the ultrasound artifacts commonly encountered in obstetrical and gynecological ultrasound including Doppler and 3D imaging artifacts.
\end{abstract}

Keywords: Ultrasound artifacts, Doppler, 3D artifacts.

How to cite this article: Zalud I, Rocha F. Artifacts, Pitfalls and Normal Variants. Donald School J Ultrasound Obstet Gynecol 2012;6(1):1-8.

Source of support: Nil

Conflict of interest: None declared

\section{INTRODUCTION}

Artifacts in ultrasound are common problem in everyday clinical practice. Differentiating real findings and deceptive artifacts is very important. A good understanding of the physical principles of ultrasound waves, equipment and their interaction with anatomy being examined is essential in distinguishing reality, normal variants and artifacts.

\section{What is the Problem?}

- What gives the multiple appearance of an intrauterine contraceptive device?

Answer: Reverberation

- Why does an early single intrauterine pregnancy sometimes look like a twin gestation?

Answer: Duplication artifact

- Why is a large cyst-like structure occasionally seen in the pelvis when it does not exist?

Answer: Mirror image artifact

- Why does a simple cyst sometimes appear to contain a sludge-like layer?

Answer: Slice thickness artifact

- Why are dermoids, even large ones, sometimes not detectable sonographically?

Answer: Shadowing (tip of the iceberg phenomenon)
- What does it all mean?

Answer: In this chapter, these intriguing artifacts are described and explained. Advice on how to recognize and in some cases, how to minimize them is also given. On the other hand, the presence of artifacts can sometimes even be helpful in clinical practice and give additional information.

\section{DEFINITION}

Artifacts in ultrasound imaging occur as structures that are one of the following:

- Not real

- Missing

- Of improper brightness

- Of improper shape

- Of improper size.

Some artifacts are produced by improper equipment operation (e.g. improper transducer location and orientation information sent to the display) or settings (e.g. incorrect receiver compensation settings). Some are caused by improper scanning technique (e.g. allowing patient or organ movement during scanning). Other as inherent in the ultrasound diagnostic method and can occur even with proper equipment and technique.

\section{MECHANISM}

Artifacts are merely errors in presentation that result from the following assumptions:

Echoes come from interfaces that are:

- Directly in front of the transducer

- At a depth equal to half the time of flight of the sound pulse multiplied by a constant velocity $(1,540 \mathrm{~m} / \mathrm{s})$.

In other words, if the pulse is reflected, refracted or otherwise affected in the body, the ultrasound machine has no way of knowing that. A blip that does not correspond to an actual interface at a corresponding point in the body may appear on the screen. The blip always appears on the screen at a point corresponding to the time since the production of the pulse and from the direction that the transducer was pointing.

\section{CLASSIFICATION}

Commonly encountered artifacts include:

- Reverberation and ring-down (comet tail)

- Shadowing 
- Enhancement

- Mirror (multipath) artifacts

- Refraction and side lobes

- Curved and oblique reflector

- Propagation speed error

- Resolution

- Doppler artifacts

- Three-dimensional artifacts.

These artifacts are seen daily. Although some of these artifacts are more pronounced in the upper abdomen, chest or neck, the examples chosen are mainly those encountered in obstetric and gynecologic ultrasound examinations.

\section{REVERBERATION}

Reverberation results in reflectors that are not real, being placed on the image. They will be placed behind the second real reflector at separation intervals equal to the separation between the first and second real reflectors. Each subsequent reflection will be weaker than prior ones. This can occur from the anterior wall of the urinary bladder, especially in an obese person (Fig. 1). The sound pulse is reflected back from the anterior wall of the bladder to the transducer face. As the transducer has to produce the true echo, it absorbs some of the pulse. However, some of that sound is reflected from the transducer-skin interface back into the body. It again hits the anterior bladder interface and is reflected back for a second time to the transducer. This produces a first reverberation artifact on the image. The ultrasound equipment assumes (incorrectly) that the signal has returned from a point in the body that is twice the distance from the transducer, as it is aware only of the time taken for the signal to return and not of the path actually travelled. This artifact is seen as a blip on the screen at twice the depth of the true echo. This is because the time taken for the first reverberation artifact is the same time taken for the pulse to

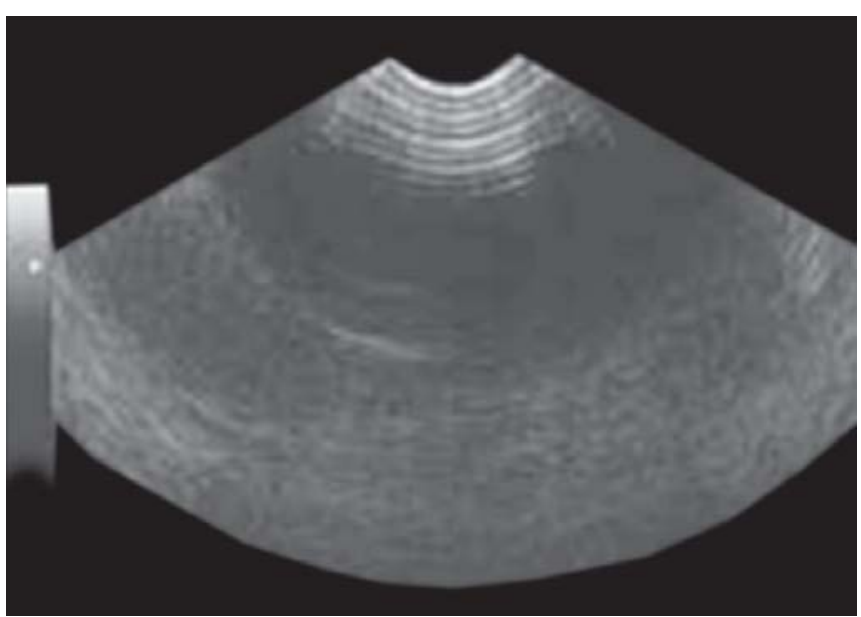

Fig. 1: Reverberation: Anterior wall of the urinary bladder in an obese person travel the original distance and back. This same reverberation can occur a second and third time to give the second or third reverberation artifact. This is commonly known as 'near-field' artifact, especially in obese patients. The echoes may be more diffuse and fuzzy if they bounce around in various directions in the subcutaneous fat before returning to the transducer. ${ }^{1}$ Occasionally, care must be taken not to confuse this artifact for an anterior placenta.

Ring-down is another type of reverberation artifact. It occurs when the sound hits a metallic structure, such as a metallic surgical clip or a group of small gas bubbles. In this situation, the sound bounces back and forth numerous times within the structure, each time sending some of the sound back to the transducer. This, therefore, appears on the screen as numerous tiny parallel echoes deep to the structure. This artifact has also been called a 'comet tail'. Certain situations are there, where there are only one or two reverberations deep to a structure. This can occur with intrauterine device (IUD) in the uterus.

Since, reverberation artifacts are produced by sound bouncing back and forth within the body, it is virtually impossible to adjust the machine to get rid of them. Although one can turn down the near gain, the real echoes will be lost along with the artifactual ones. The presence of a ring-down artifact enables the identification of gas. When this is found in an abnormal, extraluminal location, it may indicate that the patient has an abscess. In other situations, a ring-down artifact indicates that there is gas and therefore, the 'mass' seen deep to it is likely to be an artifact. When needle biopsies are done under ultrasound guidance, the needle also produces a ring-down artifact, which is particularly helpful in identifying the location of the needle on the image.

\section{SHADOWING}

Shadowing is the reduction in reflection amplitude from reflectors that lie behind a strongly reflecting or attenuating structure. Shadows in ultrasound may be due to reflection, absorption or refraction. The reflective or absorptive shadows are entirely analogous to the shadow cast by a tree in the sun. All of the light is reflected and/or absorbed by the tree trunk, so that there is a relative shadow on the tar side. With ultrasound, all of the sound beam must be blocked by a calcification to produce a shadow. There should be an echo from the near side of the structure as well. It is possible to produce an echo without a shadow if the structure only impinges on part of the sound beam without being large enough to block it completely. It is therefore possible to have small clumps of calcification that do not produce a shadow. One cannot change the size of the calcified structure. However, one can choose the correct transducer 
or the correct focal level to maximize the chance of identifying the shadow. The narrowest beam and narrowest portion of the beam are necessary to identify a shadow. If the focal depth of a transducer is adjusted either too close or too far, the echoes may be identified but not the shadows. If shadowing is not present, the calcified nature of a lesion or structure may be missed.

Air can also cause shadowing (Figs 2 and 3). Most of the time, this causes great interference on the ultrasound examination by obscuring the deeper structures. For this reason, patients have to be examined with a full bladder to displace the air-containing bowel from the pelvis. The shadow deep to gas is different from the shadow deep to a calcified structure. With the latter, although some of the sound is reflected, much of it is absorbed. With gas, the acoustic mismatch is so great that virtually all of the sound is reflected. This sound bounces around in the tissues between the transducer and the gas, and can cause numerous reverberations and other mirror image artifacts, which on the image appear deep to the echo from the gas interface. This has been called a 'dirty' shadow as opposed to the 'clean' shadow deep to bone or other calcified

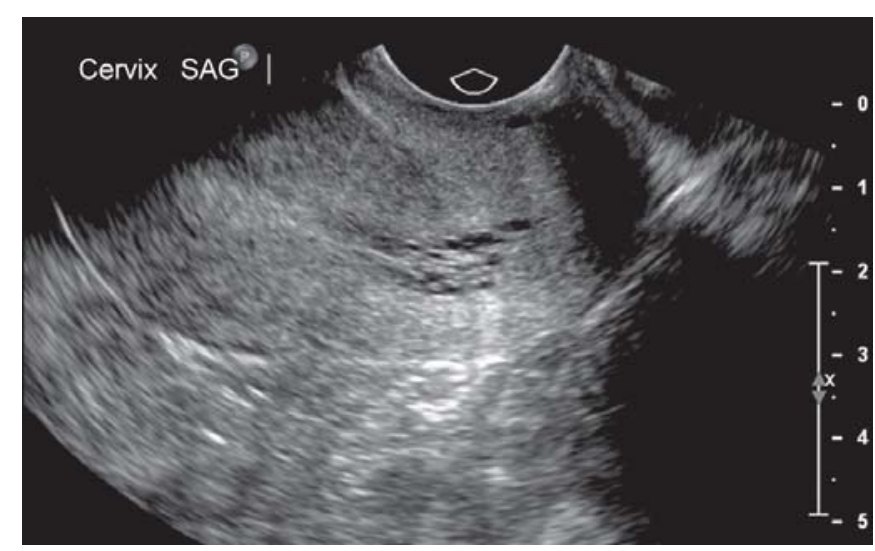

Fig. 2: Shadowing artifacts on transvaginal ultrasound caused by air in the condom. Portion of the cervix and cul-de-sac are 'in the dark'

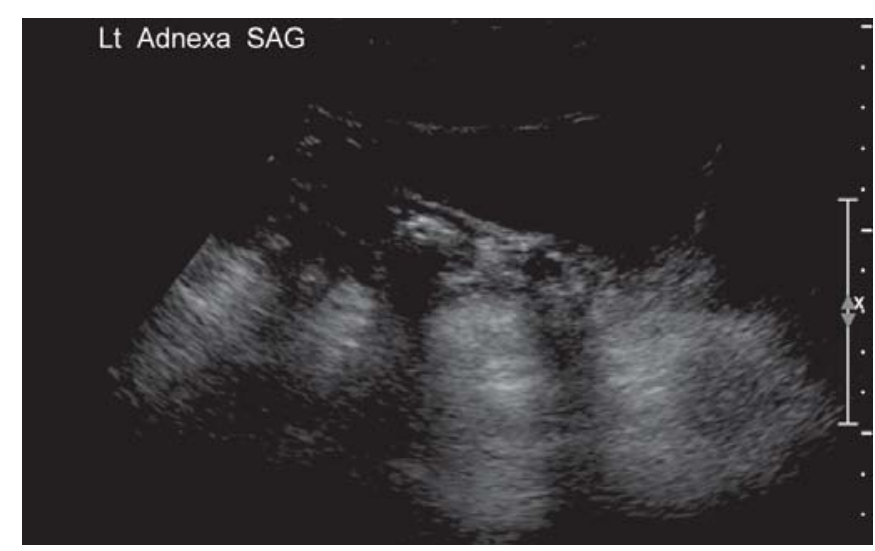

Fig. 3: Another example of shadowing caused by air in the bowels (sound-absorbing) structures. This distinction does not always hold true but most of the shadows due to gas are easily differentiated from shadows due to hard and/or calcified structures. As previously mentioned, the presence of ring-down is of further value in recognizing gas. Another type of shadowing is associated particularly with dermoid cysts. This is a peculiar situation in which there is a strong 'dirty' shadow that is likely due to the inhomogeneous structures within a dermoid. These include hair, cartilage, fat and so on. This appearance of strong shadowing can cause a difficulty in diagnosing dermoids because they can look very similar to gas and stool in the bowel, in both transverse and longitudinal scans. This is the so-called tip of the iceberg sign. ${ }^{2}$ The stool-filled rectum can mimic a dermoid or a dermoid can be overlooked by assuming that it is the rectum. When there is a clinical suspicion, a digital examination or water enema during ultrasound visualization may help differentiate between the two.

Another kind of shadow occurs at the edge of structures when the sound beam passes through an oblique interface. When the sound beam passes through a curved or oblique interface, some of the sound beam can be refracted away from the central line. This can result in a defocusing of the sound beam deep to the oblique interface and can be seen at the edge of the fetal skull, especially when the beam passes through the placenta. Occasionally, these can be at the edges of cysts in the ovaries. Refractive shadowing can also cause a drop-out of echoes deep to the bladder in the lower uterine segment or in the region of the cervix. This is especially true in patients with leiomyomas.

\section{ENHANCEMENT}

Enhancement is the opposite of shadowing. It is the increase in reflection amplitude from reflectors that lie behind a weakly attenuating structure (Figs 4 and 5). Shadowing and enhancement result in reflectors being placed on the image with amplitudes that are too low and too high, respectively. In this situation, the echoes returning from structures deep to cysts appear more intense than if the cyst were not interposed. There are two explanations for this phenomenon. One is that the fluid replaces normal soft tissue in the intervening space, decreasing its attenuation. ${ }^{3}$ The time gain compensation (TGC) is set to expect tissue between the transducer and the deepest echoes. If there is fluid instead, especially if the fluid occupies only the central portion of the image, the echoes returning from deep to the fluid collection will be more intense than expected. ${ }^{3}$ This appears as a posterior enhancement of the beam and this finding indicates that a lesion is truly cystic, even if there are internal echoes within the cyst. Occasionally, enhancement will be 


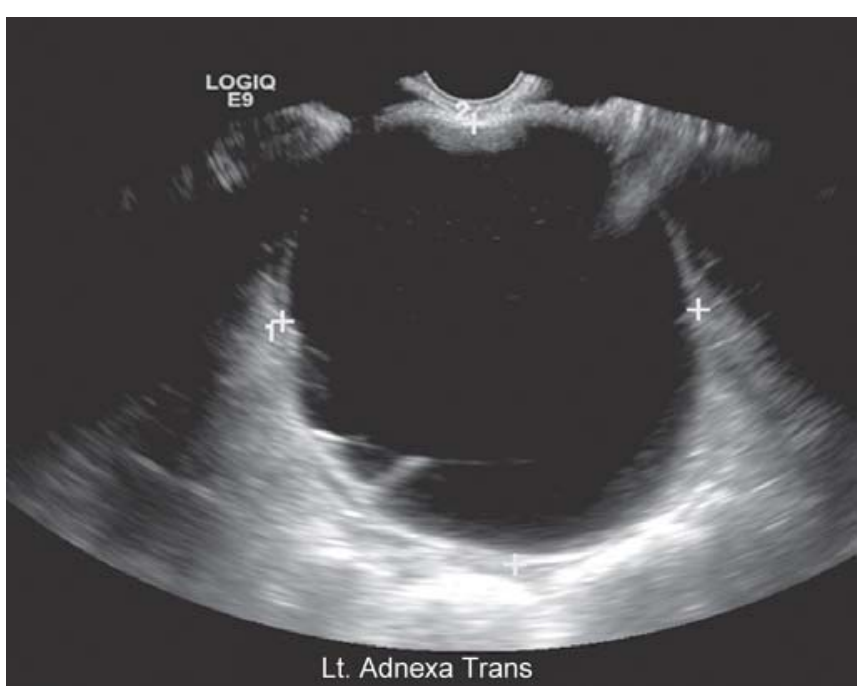

Fig. 4: Enhancement: The echoes returning from structures deep to the cyst appear more intense than if the ovarian cyst was not interposed

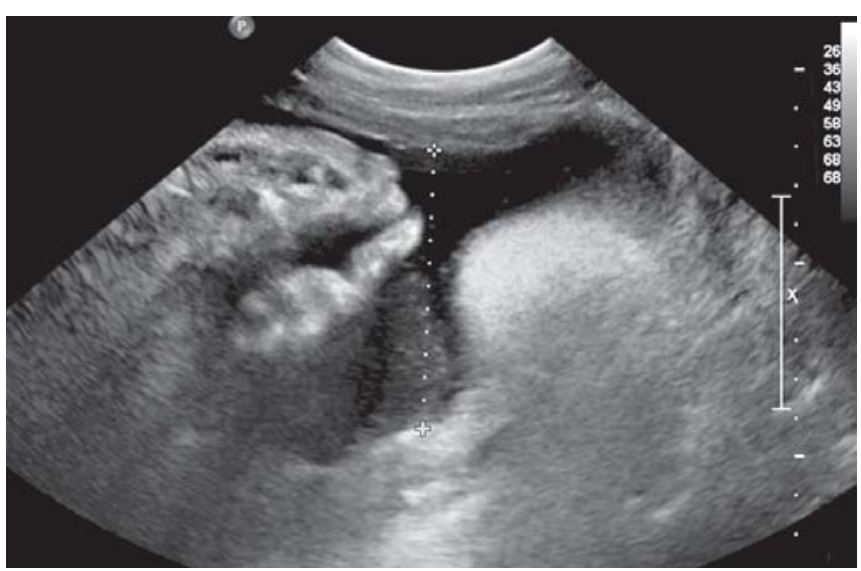

Fig. 5: Enhancement caused by amniotic fluid. Bright edges are clearly seen

noted deep to a very small cystic structure, more than what can be explained by a lack of attenuation. The small cyst acting as a lens and refocusing the sound beam may cause this enhancement. ${ }^{4}$ This is the opposite of refractive shadowing where the oblique interface defocusses the beam. Often the two coexist.

\section{MIRROR ARTIFACTS}

The term mirror or multipath artifact describes the situation in which the paths to and from a reflector are different. This artifact results in improper reflector image positioning. If separation is not sufficient, two reflectors are seen as one (missing-reflector artifact). Whereas reverberations and ring-downs are reflections that occur back and forth within the direction of the original sound beam, a mirror image artifact is one in which the sound beam is deflected away from the transducer. The reflected sound may hit a strong interface, be bounced back to the 'mirror' and then back to the transducer. The machine will therefore receive an echo and display a blip on the screen in the direction that the transducer was pointing and at a distance corresponding to the time taken. However, this will be a phantom echo since there is no interface in that position. It can also cause significant trouble when it produces a mirror image of the bladder deep to the rectum or sigmoid colon. In this situation, the phantom can closely resemble a cyst, ovarian tumor or leiomyoma. ${ }^{1}$ This kind of artifact can fool even the most experienced sonologists. Differentiating between a true lesion and a mirror image artifact can be difficult. However, the phantom cyst frequently has an unusual, somewhat triangular shape on the longitudinal scan. The back wall is often very ill defined, whereas true cystic lesions invariably have a good, clear posterior wall. It is important to realize that this artifact is seen on both transverse and longitudinal scans. One can have the patient partially empty the bladder. This will cause the phantom mass to become proportionately smaller. It is, however, important that the patient does not empty the bladder completely as real lesions can then be missed. Transvaginal scanning can be very useful in difficult cases.

\section{REFRACTION (DUPLICATION) AND SIDE LOBES}

This most interesting artifact occurs uniquely when the transducer is held in a transverse plane over the linea alba. The sound is refracted toward the midline when the transducer is pointing to the medial edge of the rectal muscle on either side. This makes small midline structures appear duplicated on the screen. This phenomenon can cause an erroneous appearance of early twins due to duplication of a single small gestational sac. In addition, intrauterine device can appear duplicated. One could similarly diagnose a bicornuate uterus erroneously. This artifact does not occur in a sagittal or transverse section once the transducer is moved to either side of the midline. ${ }^{5,6}$ Not only is the beam not as narrow as anticipated, but also there is a phenomenon called 'side lobes'. Due to refraction, there are relatively strong beams of sound outside the main beam. If one of these 'side lobes' strikes an interface and especially if that interface is concave toward the transducer, an echo is received by the transducer. Once again, the transducer and machine have no way of knowing that this came from outside the main beam and it will be displayed as though it were an interface directly in front of the transducer. These artifacts generally appear as curved lines that can be followed back to their origin. They are commonly seen in the bladder, coming off the concave surface anterior to the fundus of the uterus. Occasionally, they come from a loop of bowel that indents the bladder slightly. Refraction can cause a reflector to be improperly positioned on the display. 
A similar occurrence can be caused by reflections from side lobes. Refraction and propagation speed error can also cause a structure to be displayed with incorrect shape.

\section{OTHER ARTIFACTS}

A curved reflector can produce a reflection low in amplitude because some of the reflection is missed by the transducer. Oblique reflection can produce a reflection low in amplitude or the reflection may be completely missed by the transducer. Resolution also increases the apparent size of a reflector on a display. Propagation speed error occurs when the assumed value for propagation speed in the range equation is incorrect. Diagnostic instrumentation assumes a speed of $1,540 \mathrm{~m} / \mathrm{s}$. If the propagation speed that exists over a path traveled is greater than $1,540 \mathrm{~m} / \mathrm{s}$, the calculated distance to the reflector is too small and the display will place the reflector too close to the transducer. If the actual speed is less than $1,540 \mathrm{~m} / \mathrm{s}$, the reflector will be displayed too far from the transducer. The minimum displayed lateral and longitudinal dimensions will be the beam diameter and one-half the spatial pulse length, respectively.

\section{DOPPLER ULTRASOUND ARTIFACTS}

\section{Aliasing}

Aliasing is the most common artifact encountered in Doppler ultrasound. ${ }^{7}$ There is an upper limit to Doppler shift that can be detected by pulsed instruments. If the Doppler shift frequency exceeds one half the pulse repetition frequency (normally in the $1-30 \mathrm{kHz}$ range), aliasing occurs and improper Doppler shift information (improper direction and improper value) results. Higher pulse repetition frequencies permit higher Doppler shifts to be detected but also increase the chance of the range ambiguity artifact (Figs 6 and 7). Aliasing in a color flow system is exposed in a spatial twodimensional plane in which the aliased flow is shown in reversed color surrounded by non-aliased flow (Fig. 8). This pattern mimics the color flow appearance of separate streams in differing directions. The two patterns are, however, clearly distinguishable. In an aliased flow, the higher velocity generates a higher Doppler shifted frequency that is depicted with greater brightness. The higher the frequency shifts, the brighter the color. The brightest level in the color calibration bar (the uppermost for the flow toward the transducer and lowermost for the flow away from the transducer) represents the Nyquist limit. As the velocity and therefore, the frequency shift exceeds this limit, the color wraps around the calibration bar and appears at the other end as the most luminous color of the opposite direction. For example, a flow toward the transducer with an increasing velocity is depicted with an

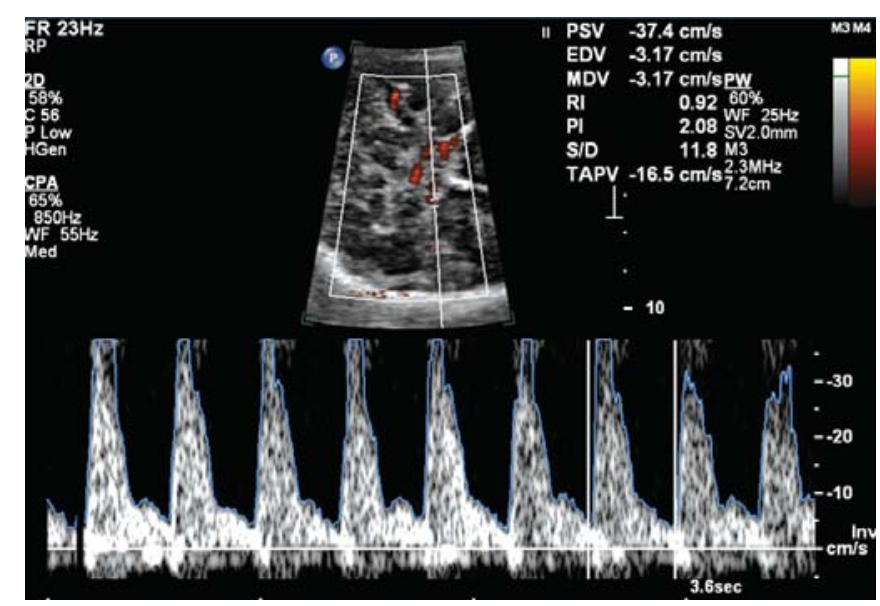

Fig. 6: Aliasing: Higher pulse repetition frequencies (PRF) permit higher Doppler shifts to be detected but also increase the chance of the range ambiguity artifact

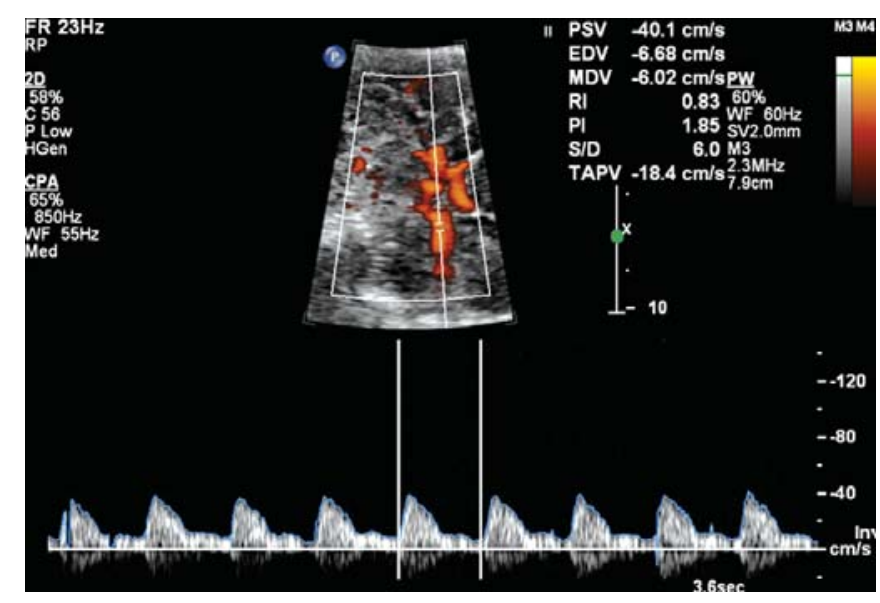

Fig. 7: Appropriate PRF setting to avoid aliasing in pulsed Doppler waveform analysis

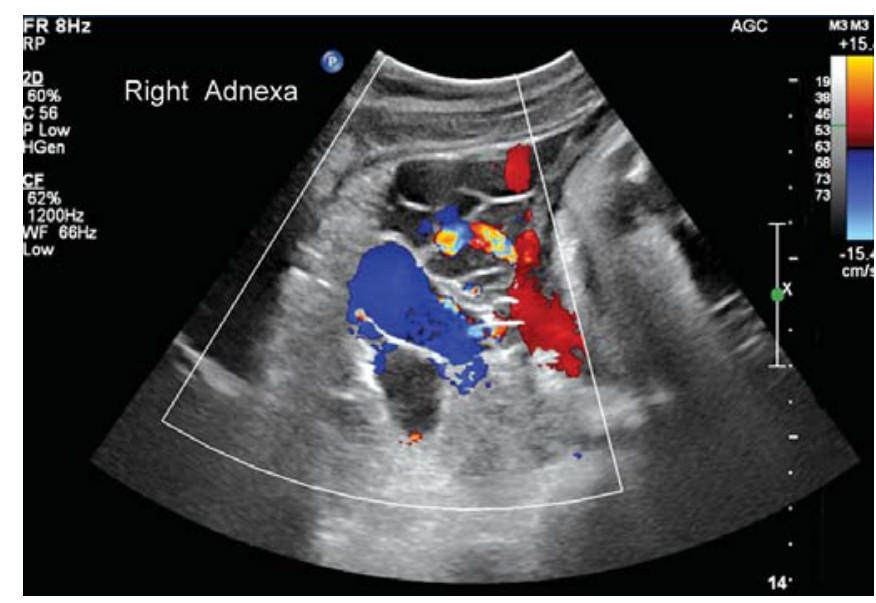

Fig. 8: Right complex, mostly cystic adnexal mass. Color Doppler aliasing shown in the middle of the mass (neovascularization) with normal red (uterine vein) and blue color (internal iliac vein) displayed on side

increasingly bright red color changing to yellow. As the Nyquist limit is reached, the color flow shows brightest yellow in the color bar and as the limit exceeds, flow is shown in the brightest blue. Thus, in an aliased flow, bright or pale color of one direction is juxtaposed against bright 
color of the opposite direction. In contrast, in genuine flow separation the distinct flow streams are depicted in the directionally appropriate colors that are separated by a dark margin. It should be noted that the hue that demarcates an aliased flow would depend on the choice of the colormapping scheme.

Aliasing can be eliminated by increasing pulse repetition frequency, increasing Doppler angle (which decreases the Doppler shift for a given flow) or by baseline shifting. The latter is an electronic 'cut and paste' technique that moves the misplaced aliasing peaks over to their proper location. It is a successful technique as long as there is no legitimate Doppler shifts in the region of the aliasing. If there are, they will get moved over to an inappropriate location along with the aliasing peaks. Other approaches to eliminating aliasing include changing to a lower frequency Doppler transducer or changing to a continuous-wave instrument. Aliasing occurs with the pulsed system because it is a sampling system. ${ }^{8}$ If samples are taken often enough, the correct result is achieved. Sufficient sampling yields the correct result. Insufficient sampling yields an incorrect result.

\section{Range Ambiguity}

In an attempt to solve the aliasing problem by increasing pulse repetition frequency, the range ambiguity problem can be encountered. ${ }^{9}$ This occurs when a pulse is emitted before all the echoes from the previous pulse have been received. When this happens, early echoes from the last pulse are simultaneously received with late echoes from the previous pulse. This causes difficulty with the ranging process. The instrument is unable to determine whether an echo is an early one (superficial) from the last pulse or a late one (deep) from the previous pulse. To avoid this difficulty, it simply assumes that all echoes are derived from the last pulse and that these echoes have originated from some depth. As long as all echoes are received before the next pulse is sent out, this will be true. However, with high pulse repetition frequencies, this may not be the case. Doppler flow information may, therefore, come from locations other than the assumed one (the gate location). In effect, multiple gates or sample volumes are operating at different depths. Instruments often increase pulse repetition frequency (to avoid aliasing) into the range where range ambiguity occurs. Multiple sample gates are shown on the display to indicate this condition. Range ambiguity in colorflow Doppler, as in sonography, places echoes (color Doppler shifts in this case) that have come from deep locations after a subsequent pulse was emitted in shallow locations where they do not belong. In practice, however, most Doppler color flow devices prevent this problem by automatically reducing the depth when the pulse repetition frequency is increased to the threshold of range ambiguity.

\section{Temporal Ambiguity}

Temporal ambiguity occurs when Doppler color flow mapping fails to depict hemodynamic events with temporal accuracy. Specifically, such a situation arises when the frame rate for color flow is too slow relative to the circulatory dynamics. As discussed earlier, the basic unit of color flow depiction is a single frame which when completed shows the average mean frequency shifts color coded and superimposed on the gray scale tissue image. The flow dynamics are, therefore, summarized for the duration of one frame. As we have noted above, the frame rate is inversely proportional to the number of scan lines and the number of samples per scan line. The slower the frame rates the better the color image quality in terms of both spatial resolution and Doppler sensitivity. Herein lies the paradox as a slower rate means longer duration of a frame. As the frame duration increases, there is a progressive loss of the ability to recognize discrete hemodynamic events.

\section{Angle of Insonation}

Angle dependency of the Doppler shifted frequencies is also a critical factor in blood-flow analysis. In sector scanning, multiple scan lines spread out from the transducer in a fanlike manner. When the sector scanner is used to interrogate a circulatory system in which the direction of flow is across these scan lines in a color window, the angle of insonation between the flow axis and the ultrasound beam changes. The angle is smallest when the flow stream enters in the sector field and progressively rises to $90^{\circ}$ as the flow approaches the center of the field. Concurrently, the Doppler shifted frequencies progressively decline and may become undetectable at the center of the color field. A sector scanner may also create apparently contradictory directional information in a vessel traversing across the color field. As the flow approaches the midline of the field, the flow is depicted in color encoding for flow toward the transducer which usually is red; as the flow moves away, it will be encoded blue. Thus, the same vessel will show bidirectional flow. This paradox actually highlights the basic concept of representation of flow directionality by any Doppler system.

\section{Doppler Mirror Artifact}

The mirror image artifact can also occur with Doppler systems. This means that an image of a vessel and a source of Doppler shifted echoes can be duplicated on the opposite 
side of a strong reflector (such as a bone). The duplicated vessel containing flow could be misinterpreted as an additional vessel. It will have a spectrum similar to that for the real vessel (Fig. 9). A mirror image of a Doppler spectrum can appear on the opposite side of the baseline when, indeed, flow is unidirectional and should appear only on one side of the baseline. ${ }^{10}$ This is an electronic duplication of the spectral information. It can occur when receiver gain is set too high (causing overloading in the receiver and cross talk between the two flow channels) or with low gain (where the receiver has difficulty determining the sign of the Doppler shift). It can also occur when Doppler angle is near $90^{\circ}$. Here the duplication is usually legitimate and this is because beams are focused and not cylindrical in shape. Thus, portions of the beam can experience flow toward while other portions can experience flow away.

\section{D ULTRASOUND ARTIFACTS}

Three-dimensional (3D) ultrasonography is a rapidly developing area with increased application in obstetrics and gynecology. Unfortunately, its technology is not only susceptible to artifacts, but the volume acquisition also can present unique artifacts. The images obtained are usually acquired by a series of $2 \mathrm{D}$ image planes that are rendered to a volume 3D image. Motion or vibration of the targeted organ during the acquisition of a volume introduces a motion artifact into the volume. The motion artifact affects the overall quality of a volume and is particularly relevant in obstetric imaging because of the movement of the fetus. ${ }^{11}$ Shadowing from adjacent structures can reproduce, for example, an apparent limb defect or cleft lip and palate (Fig. 10). ${ }^{12}$

Technical aspects of imaging must be considered as this new technology is learned by practitioners in our field. Not only are there artifacts inherent to 2D imaging present in 3D ultrasound but additional artifacts specific to volume

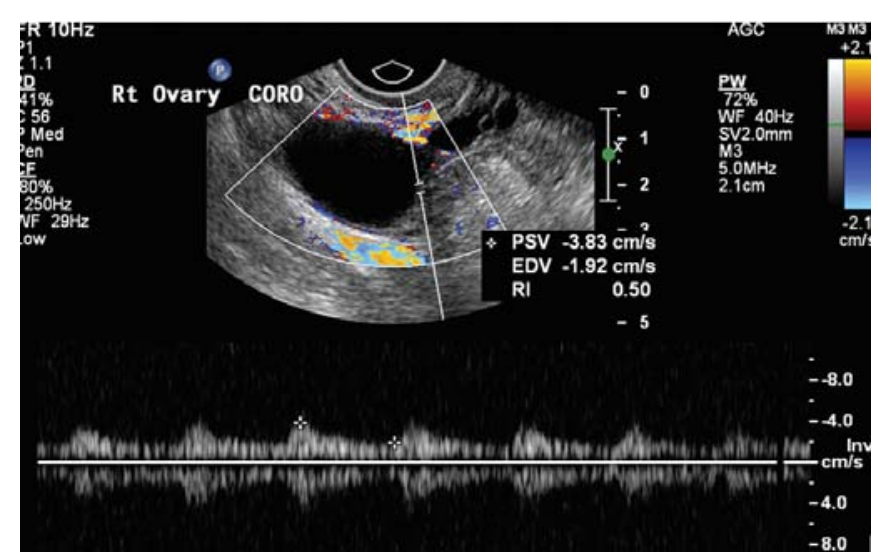

Fig. 9: Mirror effect: A mirror image of a Doppler spectrum appeared on the opposite side of the baseline when blood flow was unidirectional and should appear only on one side of the baseline

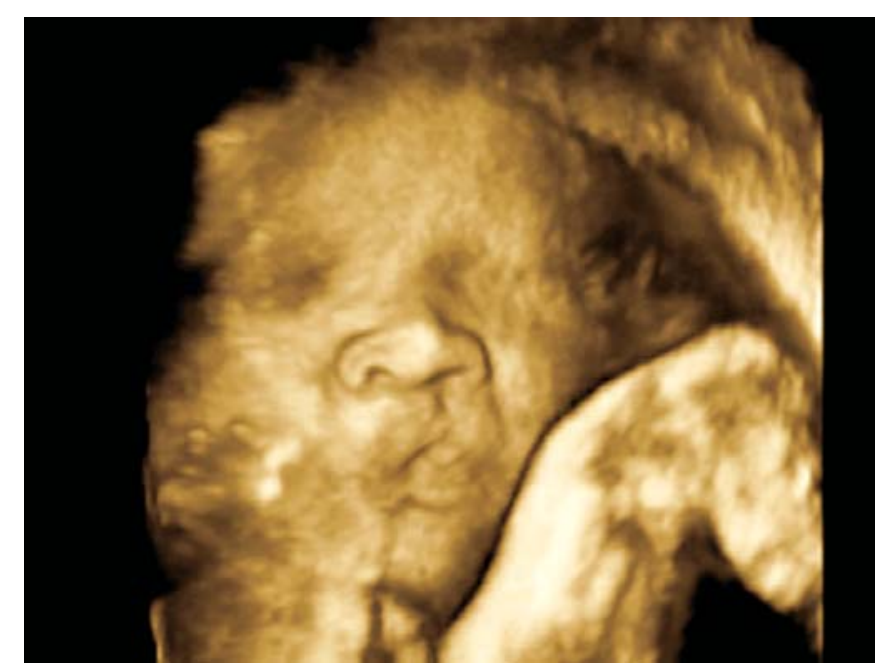

Fig. 10: 3D artifact: Shadowing from adjacent structures reproduced an apparent cleft lip and palate

imaging have also emerged. Such acoustic artifacts as dropout and shadowing which are well known to the ultrasound community are present in 3D imaging, although more difficult to recognize due to different and unfamiliar displays. Color and power Doppler artifacts relating to gain and flash may also be confusing in rendered images. Threedimensional volume sets are hampered by fetal movement, cardiac motion, as well as movement of adjacent structures. Acoustic shadowing and other artifacts look very different when displayed in 3D volumes and may be more difficult to recognize than on standard 2D due to lack of specific training of personnel. Acquiring data from multiple orientations may avoid artifacts of this type.

\section{CONCLUSION}

A prerequisite for optimal utilization of ultrasound in obstetrics and gynecology is an in-depth knowledge of the principles and limitations of this dynamic technique. It is important to appreciate that the appearance of Doppler images is influenced by the operational setting of the equipment that must be taken into account for any reliable interpretation. Only persons with sufficient training and education should perform diagnostic ultrasound. One of the major reasons for so many conflicting and controversial results in the ultrasound literature originates from technique complexity and rather limited education in physics and technique. With all artifacts, but especially with mirror image artifacts, it is important not to let a superficial knowledge cause trouble. Once the cause and nature of an artifact are understood, it is important not to misinterpret a real lesion as an artifact and miss the true pathology. This can happen, particularly with pelvic masses, such as leiomyomas with poor through transmission in which the deep wall is not well seen. If there is also an artifact situated 
near where the deep wall would be, the actual mass might be dismissed as simply an artifact. One must pay attention at all times not only to identify artifacts, but also not to let them interfere with the identification of true lesions. While the more common artifacts seen on ultrasound images frequently are ignored and appreciated as such, it is certainly interesting to know why they occur. On the other hand, the usefulness of artifacts cannot be underestimated. Occasionally, the identification of an artifact may prevent the novice from making an important error in diagnosis or management. An appreciation and understanding of how to avoid artifacts can help even the more experienced practitioner decide whether a structure is real. It is also important not to ignore real pathology under the assumption that it is caused by an artifact.

\section{REFERENCES}

1. Laing FC, Brown DL, DiSalvo DN. Gynecologic ultrasound. Radiol Clin North Am 2001;39(3):523-40.

2. Guttman PH. In search of the elusive benign cystic ovarian teratoma: Application of the ultrasound 'tip of the iceberg' sign. J Clin Ultrasound 1977;5(6):403-06.

3. Filly RA, Sommer FG, Minton MJ. Characterization of biological fluids by ultrasonic computed tomography. Radiology 1980;134(1):167-71.

4. Robinson DE, Wilson LS, Kossoff G. Shadowing and enhancement in ultrasonic echograms by reflection and refraction. J Clin Ultrasound 1981;9(4):181-86.
5. Buttery B, Davison G. The ghost artifact. J Ultrasound Med 1984;3(2):49-52.

6. Sauerbrei EE. The split image artifact in pelvic ultrasonography: The anatomy and physics. J Ultrasound Med 1985;4(1):29-34.

7. Zalud I, Kurjak A, Maulik D, et al. Transvaginal Doppler: measurements and errors. In: Kurjak A, Kupesic S (Eds): An atlas of transvaginal color Doppler. New York-London, Parthenon Publishing 2000;255-62.

8. Mitchell DG. Color Doppler imaging: principles, limitations, and artifacts. Radiology 1990;177(1):1-10.

9. Kremkau FW. Doppler color imaging: Principles and instrumentation. Clin Diagn Ultrasound 1992;27:7-60.

10. Burns PN. Principles of Doppler and color flow. Radiol Med 1993;85(5 Suppl 1):3-16.

11. Nelson TR, Pretorius DH, Hull A, et al. Sources and impact of artifacts on clinical three-dimensional ultrasound imaging. Ultrasound Obstet Gynecol 2000;16(4):374-83.

12. Hull AD, Pretorius DH, Lev-Toaff A, et al. Artifacts and the visualization of fetal distal extremities using three-dimensional ultrasound. Ultrasound Obstet Gynecol 2000;16(4):341-44.

\section{ABOUT THE AUTHORS}

\section{Ivica Zalud (Corresponding Author)}

Professor and Chief, Division of Maternal Fetal Medicine, Department of Obstetrics and Gynecology and Women's Health, John A Burns School of Medicine, University of Hawaii, Honolulu, Hawaii, USA, e-mail: ivica@hawaii.edu

\section{Frederico Rocha}

Clinical Instructor, Division of Maternal Fetal Medicine, Department of Obstetrics and Gynecology and Women's Health, John A Burns School of Medicine, University of Hawaii, Honolulu, Hawaii, USA 